\title{
Vom Sprechenkönnen und Gehörtwerden
}

\author{
Einleitung in den Schwerpunkt „Partizipation und Selbstorganisation \\ in der Wohnungslosenhilfe"
}

Seit Jahren wird von und in der Sozialen Arbeit Ressourcenorientierung statt Defizitorientierung gefordert. Empowerment und die Ermöglichung von erlebter Selbstwirksamkeit (Agency) sind begründete und weithin anerkannten Orientierungen für die Praxis. Es geht um den Abbau paternalistischer Strukturen, um parteiliches Miteinander und um Partizipation. Verglichen mit der Jugendhilfe und der Eingliederungshilfe scheint die Wohnungslosenhilfe ein wenig spät dran zu sein. Aber auch in diesem traditionellen Feld der Sozialen Arbeit hat sich in den letzten Jahren etwas getan.

U nser Schwerpunkt beginnt mit einer Besprechung und Evaluation relevanter Ansätze, die es bisher auf europäischer Ebene gegeben hat, angefangen mit der Entwicklung von ETHOS (European Typology of Homelessness and Housing Exclusion), einer umfassenden Typologie von Wohnungslosigkeit, die durch den Europäischen Dachverband FEANTSA (Fédération Européenne d'Associations Nationales Travaillant avec les Sans-Abri) erstellt wurde.

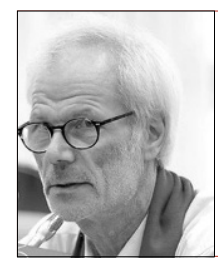

\section{Peter Herrmann $n^{1,3}$}

${ }^{1}$ Changsha, Volksrepublik China

${ }^{3}$ Hochschule für Wirtschaft und Recht Berlin, Berlin, Deutschland

Dr., Professor für Human Rights.

herrmann@esosc.eu

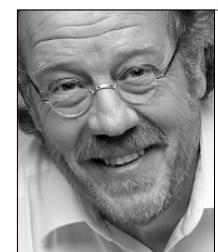

\section{Peter Szynka²}

${ }^{2}$ Bremen, Deutschland

*1954. Dr., Studium der Sozial- und Sozialarbeitswissenschaft. Bis 2018 Referent für Wohnungslosenhilfe beim Diakonischen Werk evangelischer Kirchen in Niedersachsen e. V., seit 2020 Verwalter der Professur für "Sozialarbeitswissenschaft, Gemeinwesenarbeit und Community Organizing" an der Hochschule Hannover.

szynka@uni-bremen.de

Zusammenfassung Dienste allgemein und speziell die Dienste für Wohnungslose sind immer auch dadurch gekennzeichnet, dass es Kommunikationsschwierigkeiten zwischen den unterschiedlichen Akteur_innen gibt. Diese Einleitung in den Schwerpunkt „Wohnungslosigkeit“ problematisiert diesen Umstand und weist nicht zuletzt darauf hin, dass alle Beteiligten den gesellschaftlich gleichen Status als Bürger haben.

Schlüsselwörter Wohnungslosigkeit, Soziale Dienste, Partizipation, (Nicht-) Verstehen
Weiterhin wird die „Housing-First“-Strategie beschrieben, mit der die Wohnungsversorgung wieder an die erste Stelle der Bemühungen gerückt ist. Außerdem wird das FEANTSA-Toolkit zur Partizipation vorgestellt, das ähnlich wie in der Gemeinwesenarbeit Betroffene als „Experten ihrer Lebenswelt" anerkennt und einbezieht (Josef Finnerty).

In Deutschland wurde das Thema Partizipation von der BAG-W (Bundesarbeitsgemeinschaft Wohnungslosenhilfe e. V.) aufgegriffen. Unter Mitwirkung der BBI (Bundesbetroffeneninitiative) und des Armutsnetzwerks e. V. wurden Regelungen für die Beteiligungen von Betroffenen an Tagungen und Gremien getroffen und ein richtungsweisendes Positionspapier entwickelt. Die von Peter Szynka vorgelegten Thesen sind eine Erweiterung und Aktualisierung seines Beitrags auf einer BAG-Tagung, die im Jahr 2010 stattgefunden hat.

Der Beitrag von Stefan Schneider berichtet von den Wohnungslosentreffen, die in Deutschland seit 2016 regelmäßig und bundesweit (mit Gästen aus dem übrigen Europa) stattgefunden haben und aus der die Selbstorganisation Wohnungsloser e. V. hervorgegangen ist. Bei den Wohnungslosentreffen handelt sich hier um das bisher umfangreichste Projekt zum Thema Partizipation wohnungsloser Menschen, das in der Bundesrepublik stattgefunden hat. Es steht in der Tradition früherer Treffen in Stuttgart (1929 und 1981) und Uelzen (1991) und greift die Forderungen der BAG-W und von FEANTSA nach mehr zielgruppenspezifischer Partizipation auf (s. Abb. 1).

Abschließend berichten Alexander Eisele u. a. von ihrem kooperativen Forschungsprojekt zur Akzeptanz ordnungsrechtlicher Unterkünfte in Hannover. Sie haben dafür eine vom Evangelischen Bundesfachverband Exis- 


\section{Durchblick: Partizipation in der Wohnungslosenhilfe}

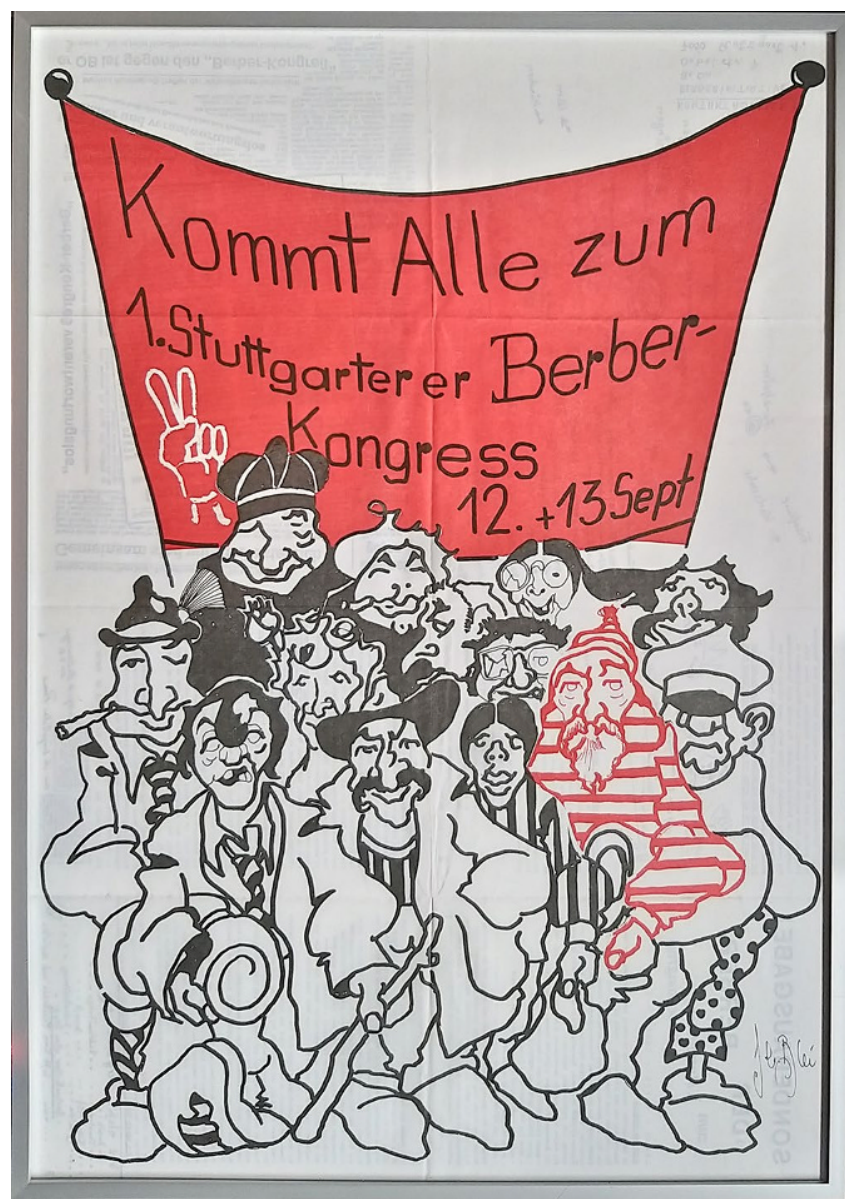

Abb. 1 Plakat zum Stuttgarter Berber-Kongress 1981; @ Peter Szynka

tenzsicherung und Teilhabe e. V. (EBET) in Auftrag gegeben Lebenslagenstudie unter konsequenter Einbeziehung „erfahrener Forschungsmitglieder“, also Menschen, die wohnungslos sind oder waren, weitergeführt und auf die örtliche Situation der Stadt Hannover angewandt.

\section{Mühen der Ebene}

Die Beiträge befassen sich mehr oder weniger mit den akzeptierten Forderungen zur Verbesserung der Lebenslage der Betroffenen und den hiermit verbundenen „Mühen der Ebene“. Um den Stand der Praxis zu erfassen und zu bewerten, greifen wir deshalb die berühmte Frage „Können die Nachgeordneten sprechen?“ aus der postkolonialen Theorie auf, die von G.C. Spivak formuliert wurde. Bei der Beantwortung geht es Spivak ganz allgemein um Bedingungen subalterner Artikulation. Nachgeordnet bzw. subaltern ist hierbei ein Sammelbegriff für Benachteiligte aller Art. Es können Menschen mit niedrigerem Status sein, Angehörige niederer Kasten, Exkludierte, Menschen ohne Bildung, befristet Beschäftigte und eben auch Einwanderer und Wohnungslose. Es geht damit ganz allgemein um die Sprach- und Sprechmöglichkeiten dieser Menschen, in unseren Bei- trägen eben um die der Wohnungslosen. Wie können wir Sprech- und Interaktionsordnungen so verändern, dass wohnungslose Menschen nicht nur nachgeordnet sind? Es handelt sich um eine Frage, die bei der Diskussion um gerechte Wohnungsversorgung, Planung sozialer Unterstützungsleistungen, Qualität sozialer Dienste, Bestandsaufnahmen und Statistiken eigentlich unumgänglich ist. Fragen der Gerechtigkeit können nicht ohne die Beteiligung derer abschließend behandelt werden, die ungerecht behandelt werden oder wurden. Das gilt also nicht nur, aber gerade auch für wohnungslose Menschen.

Die Erfahrungen aus der Selbstorganisation Wohnungsloser und den kooperativen Forschungsprojekten zeigen eindeutig, dass die Wohnungslosen sehr wohl sprechen können. Auch, dass es wert ist, ihnen gut zuzuhören. Anders ausgedrückt: Die Wohnungslosen haben etwas Wichtiges beizutragen und Wertvolles zu sagen. Sie sind prinzipiell in der Lage, dieses auch zu tun. Schneider und Eisele weisen aber eindrücklich darauf hin, dass dies nicht nur eine Sache der Interessen, sondern auch eine der Ressourcen ist. Die Fragen, die sich im internationalen Diskurs auch an die Frage von C.G. Spivak angeschlossen hat und die wir deshalb auch hier stellen müssen, ist aber: „Werden die Nachgeordneten auch gehört?". Es ist zu betonen, dass es hier nicht nur um die Betroffenen selbst geht, die ihre Anliegen individuell, etwa im Sozialamt, bei der Wohnungs- und Arbeitssuche vortragen, sondern auch um die Artikulation von Bedürfnissen im öffentlichen Raum durch eigene Interessensgruppen, wie etwa der Wohnungslosenselbsthilfe e. V. oder durch Organisationen, die ihre vermeintlichen Interessen mitvertreten.

\section{Aktive Beteiligte}

Auch hier lassen sich Anzeichen dafür finden, dass durch die Aktivitäten der letzten Jahre vielfältiges Interesse geweckt worden ist: Die Beteiligten werden von Pressevertretern angefragt, zu Anhörungen und Konferenzen eingeladen, haben gelernt, sich auch online oder im Rundfunk zu artikulieren. Sie mischen sich ein und laden sich zu verschiedenen Events selbst ein. Offen ist nur, wieweit dieses bisher nur punktuell in Erscheinung tretende Interesse von Medien und Politik trägt und wieweit die vorhandenen persönlichen und materiellen (Projekt-) Ressourcen wirklich tragen werden. Die entscheidende Frage muss daher hier wie auch in anderen Fällen lauten „Werden die Nachgeordneten verstanden? “ oder, präziser, „Nehmen die Entscheidungsträger die Bedürfnisse und Forderungen wohnungsloser Menschen genügend zur Kenntnis und sind sie willens und bereit ihrer Verantwortung auf dem Weg zu einer gerechteren Gesellschaft nachzukommen “. Auch die vorgestellten Projekte gezeigt haben, dass Verständigung grund- 
sätzlich möglich, sinnvoll und unverzichtbar ist, sind wir jedoch noch weit entfernt von einer allgemeinen Kultur der Verständigung im Bereich der Wohnungslosenhilfe.

Mehr noch: auch wenn es auf den ersten Blick banal erscheinen mag, so ist doch nicht immer klar, in welcher Angelegenheit, mit welcher Agentur und mit welchem Ziel Verständigung betrieben wird. Man könnte man die Wohnungslosen auch anhören, ihre Anträge und Vorschläge ablehnen, oder sie diskreditieren und Politik gegen sie machen. Aber um dieses Extreme geht es gar nicht. Tatsächlich sind die Kriterien für jegliche Verständigungssituation sehr unterschiedlich. Man muss fragen, ob die Anhörenden überhaupt die Sprache der Angehörten sprechen und verstehen. Ganz offensichtlich ist dies nicht immer der Fall, wenn etwa die Hilfestellen und Agentur der Meinung, es gehe ausschließlich darum, zuallererst eine Wohnung zu finden, während die Hilfesuchenden nicht nur und vielleicht nicht einmal primär ein Dach über dem Kopf suchen, sondern einen Ort, an dem sie bleiben können, bis sie eine Wohnung finden.

Die BAG-W kennt dafür folgende Varianten: das Schlafen auf der Straße, Provisorien wie Zelt, Wohnmobil, Abrisshaus, Biwak o. ä., Notunterkünfte, die nur nachts geöffnet sind, ohne Untermietvertrag bei Freund_innen und Bekannten, im Wohnheim, Hostel, ordnungsrechtliche Unterkünfte, betreutes Wohnen, stationäres Wohnen (Heime), betreute Wohngemeinschaften, betreutes Einzelwohnen ... Wie diese Zeit überbrückt werden soll und wie aber dieser Ort aussehen soll, sollte Gegenstand von Verständigungsprozessen sein, bei denen die Wohnungslosen gehört werden und bei denen auf ihre spezifischen Bedürfnisse eingegangen wird. Dann stellt sich die nächste Frage: Sind diejenigen, die zuhören, überhaupt in der Position, in der sie auf das Gehörte tatsächlich eingehen können? Das System in Deutschland, vor allem auch die ambulante Arbeit, ist schon so stark differenziert, dass auf das Gehörte nur die Antwort folgen kann: wir können nichts tun, der Wohnungsmarkt gibt das nicht her! Im günstigsten Fall werden die Personen zu einer kompetenteren Stelle weitergeleitet. In den meisten Fällen finden sie aber auch da keine Hilfe. Den Aufenthalt in Obdachlosenunterkünften, Heimen und betreuten Wohnformen empfinden die einen zunächst als Entspannung, für andere bedeutet das aber oftmals auch Bevormundung, Einschränkung und Regulierung.

\section{Organisierte Bürger}

Auch die Helfenden sind eingespannt in die Bedingungen und Anforderungen eines Systems, das die Probleme erst verursacht hat. Das genaue Lesen der Beiträge kann somit auch ein Paradox aufdecken. Oftmals wird den Hilfsorganisationen vorgeworfen, dass sie eine zu komplizierte Sprache sprechen und den Hilfesuchenden fremd gegenübertreten. Aber bereits der Begriff der „Hilfesuchenden“ verweist auf ein reduziertes Verständnis auf Seiten der Anbieter. Vor vielen Jahren wurde im Rahmen eines EU-Projekts zur „Zugänglichkeit inklusiver Sozialdienste“ (s. Herrmann 2007), der Begriff des „organisierten Bürgers“ geprägt. Er lehnt sich an Antonio Gramscis Begriff des „organischen Intellektuellen“ an, der im Gegensatz zum „traditionellen Intellektuellen“ Kontakt zur Lebenswirklichkeit der Menschen hat, für die er spricht und der in der Lage ist, mit den „traditionellen Intellektuellen“, die es ja immer noch gibt, und zwar an entscheidenden Stellen, auf Augenhöhe zu verhandeln. Diese Rolle einzunehmen sollte auch „Bürgern ohne Wohnung“ möglich sein.

Innerhalb eines Organisationssystems sollte das Ziel darin bestehen, allen Mitgliedern (in traditionellen Begriffen: den Hilfsempfangenden, den Helfenden, den Verwaltenden) den Status von organisierten Bürgern im besten Sinne des Wortes zukommen zu lassen. Und der eigentliche Sinn der Rede vom „organisierten Bürger" besteht darin, dass nicht einer nur zuhört und der andere nur spricht, sondern dass sich vielmehr alle Teilnehmenden in einem Diskurs befinden.

Eingegangen. 27. Januar 2021

Angenommen. 27. Januar 2021

Funding. Open Access funding enabled and organized by Projekt DEAL.

Open Access. Dieser Artikel wird unter der Creative Commons Namensnennung 4.0 International Lizenz veröffentlicht, welche die Nutzung, Vervielfältigung, Bearbeitung, Verbreitung und Wiedergabe in jeglichem Medium und Format erlaubt, sofern Sie den/die ursprünglichen Autor(en) und die Quelle ordnungsgemäß nennen, einen Link zur Creative Commons Lizenz beifügen und angeben, $o b$ Änderungen vorgenommen wurden.

Die in diesem Artikel enthaltenen Bilder und sonstiges Drittmaterial unterliegen ebenfalls der genannten Creative Commons Lizenz, sofern sich aus der Abbildungslegende nichts anderes ergibt. Sofern das betreffende Material nicht unter der genannten Creative Commons Lizenz steht und die betreffende Handlung nicht nach gesetzlichen Vorschriften erlaubt ist, ist für die oben aufgeführten Weiterverwendungen des Materials die Einwilligung des jeweiligen Rechteinhabers einzuholen.

Weitere Details zur Lizenz entnehmen Sie bitte der Lizenzinformation auf http://creativecommons.org/licenses/by/4.0/deed.de

\section{Literatur}

Herrmann, P. (2007). Accessibility of social services-a general matter of assessing services in a social quality perspective. In P. Hermann, A. Brandstetter \& C. O'Connel (Hrsg.), Defining social services in Europe-between the particular an the general (S. 156-176). Baden-Baden: Nomos. 